\title{
The impact of improved plasma diagnostics on modeling the X-ray Universe
}

\author{
Junjie Mao, ${ }^{a, b, 1}$ François Mernier, ${ }^{c, d, b}$ Jelle S. Kaastra, ${ }^{b, e}$ Liyi Gu, ${ }^{f, b}$ Missagh Mehdipour, ${ }^{b}$ \\ and Jelle de Plaa $^{b}$ \\ ${ }^{a}$ Department of Physics, University of Strathclyde, Glasgow G4 ONG, UK \\ ${ }^{b}$ SRON Netherlands Institute for Space Research, Sorbonnelaan 2, 3584 CA Utrecht, the Netherlands \\ ${ }^{c}$ MTA-Eötvös University Lendület Hot Universe Research Group, Pázmány Péter sétány 1/A, Budapest, \\ 1117, Hungary \\ ${ }^{d}$ Institute of Physics, MTA-Eötvösy, Pázmány Péter sétány 1/A, Budapest, 1117, Hungary \\ ${ }^{e}$ Leiden Observatory, Leiden University, PO Box 9513, 2300 RA Leiden, the Netherlands \\ ${ }^{f}$ RIKEN High Energy Astrophysics Laboratory, 2-1 Hirosawa, Wako, Saitama 351-0198, Japan \\ E-mail: junjie.mao@strath.ac.uk
}

\begin{abstract}
Aвstract: The high-resolution X-ray spectrum of the Perseus galaxy cluster observed with the Hitomi satellite challenges astrophysical collisional ionized plasma models that are widely used in the community. Although Hitomi spun out of control, several Hitomi-level missions have been proposed and some funded. The spectrometers aboard these future missions have a broader energy range and/or a higher spectral resolution to achieve different scientific goals. Accurate plasma models and atomic data are crucial for plasma diagnostics of high-quality spectra. Here, we present a few cases where improvement of plasma diagnostics will be decisive to better understand celestial bodies and their physical processes at play. We focus on collisional ionized and photoionized astrophysical plasmas in the context of developments of plasma models, as well as current and future generations of spectrometers.
\end{abstract}

Keywords: Plasma diagnostics - probes; Data analysis; Spectrometers; X-ray detectors and telescopes

\footnotetext{
${ }^{1}$ Corresponding author.
} 


\section{Contents}

1 Hot plasmas in the Universe $\quad 1$

2 Elemental abundance of intracluster media $\quad 2$

3 Density of photoionized outflows 4

4 Future perspectives $\quad 5$

\section{Hot plasmas in the Universe}

Hot plasmas can be found in a wide range of astrophysical environments. For instance, stellar coronae, the accretion flow of the supermassive black holes at the center of nearly all galaxies, the intracluster media among member galaxies within galaxy clusters, and the warm-hot intergalactic media (i.e., the cosmic web filaments) among clusters of galaxies. In fact, about half of the baryons in the entire Universe are expected to be in the form of hot plasmas with a temperature of millions to billions of degree and shine in the X-ray wavelength range (0.1-100 $⿱$ ). That is to say, X-ray observational facilities hold the key to probe the nature of these hot plasmas.

Spectroscopy, as one of the main techniques to study the electromagnetic radiation of the Universe, is used to constrain physical properties (temperature, density, chemical composition, microscopic turbulence, line of sight velocity, etc.) of the observing targets. Plasma diagnostics in the X-ray band provide important information to answer major astrophysical questions involving hot plasmas like "How does AGN feedback influence the host galaxies?", "What is the chemical composition of the Universe and what is the origin of the observed elements?", and "Where are the missing baryons in the local Universe?".

In 1975, the very first X-ray spectrum $(1.3-16 \mathrm{keV})$ of the Perseus galaxy cluster was obtained with the collimated proportional counter aboard Ariel 5. As shown in [1], a broad emission feature peaking around $\sim 7 \mathrm{keV}$ stands out above the continuum. This emission feature was interpreted as (unresolved) transitions from H-like and He-like Fe ions, while the continuum was described as the thermal bremsstrahlung emission of the hot intracluster medium. About four decades later, the Perseus galaxy cluster was revisited by Hitomi (previously known as ASTRO-H [2]). The Soft $\mathrm{X}$-ray Spectrometer (SXS) aboard Hitomi achieves an unprecedented energy resolution of $5 \mathrm{eV}$ throughout its soft X-ray band. As a result, the "single" emission feature observed with Ariel 5 in the $5-8 \mathrm{keV}$ is resolved as multiple emission lines: a prominent $\mathrm{He} \alpha$ triplet from Fe XXV $(\sim 6.5-6.6 \mathrm{keV})$, the Ly $\alpha$ line from Fe XXVI $(\sim 6.8-6.9 \mathrm{keV})$ and $\mathrm{He} \beta$ triplet from Fe XXV (around $\sim 7.75 \mathrm{keV}$ ), several weak emission lines from highly ionized Fe ions, one weak emission feature from Ni XXVII, even weaker fluorescence lines of neutral $\mathrm{Fe} \mathrm{I}$ and $\mathrm{He} \alpha$ triplets from $\mathrm{Cr}$ XXIII and Mn XXIV [3]. 
Advance in technology certainly provides a better view of the hot plasmas in the Unvierse [4]. Benchmarking the Hitomi/SXS spectrum of the Perseus galaxy cluster with collisional ionized plasma models widely used in the community reveals that accurate plasma models and the underlying atomic data are crucial to determine the physical properties (temperature, emission measure, and abundance, etc.) of the intracluster medium of Perseus. That is to say, improvement of plasma models and atomic data can exert an impact on our understanding of the X-ray Universe. In the following, we focus on two examples, elemental abundance of intracluster media and density of photoionized outflows, in the context of outdated and updated plasma models, as well as current and future generations of spectrometers.

\section{Elemental abundance of intracluster media}

A galaxy cluster typically consists of hundreds of member galaxies ${ }^{1}$, with a total mass of $10^{14-15} \mathrm{M}_{\odot}$. A large fraction $(\sim 15-20 \%)$ of the mass is in the form of hot plasma with $k T \sim 10^{7-8} \mathrm{~K}$, namely the intracluster medium (ICM), while the visible member galaxies contribute less than $\sim 5 \%$ [5-7]. The rest of the mass is in the form of dark matter.

A typical X-ray spectrum of the ICM in a relaxed cluster contains emission lines from various elements (e.g., N, O, Mg, Si, S, Fe, and $\mathrm{Ni}$ ). These elements are originally synthesized in various types of stars within the cluster:

- Asymptotic giant branch (AGB) stars are low- and intermediate mass stars with $M_{*} \in$ $(0.9,7) \mathrm{M}_{\odot}$ undergoing the last nuclear burning phase. In this phase, the stellar envelope is enriched with products of hydrogen and helium burning, as well as heavy elements produced by the slow neutron capture process [8]. These elements are eventually expelled into the interstellar medium (ISM) via a slow stellar wind.

- Massive stars in the mass range of $M_{*} \in(11,140) \mathrm{M}_{\odot}$ undergo Fe core collapse at the end of their stellar evolution [9]. If the entire star does not collapse into a black hole with no mass ejection, it explodes as core-collapse supernovae ( $\mathrm{SNcc}$ ) and release elements into the ISM.

- Degenerate stars in a binary system also contribute to the chemical enrichment of the ISM, mainly via Type Ia supernovae (SNIa) [9].

Throughout the evolution of the galaxy cluster, different transportation mechanisms working on different size-scales and time-scales redistribute these elements from the small-scale ISM into the large-scale ICM [5]. Different enrichment channels (AGBs, SNcc, and SNIa) leave specific abundance patterns so that the observed (time-integrated) ICM elemental abundance can be used to infer the origin of these elements, as shown in Figure 1.

Abundances are measured by fitting the observed spectrum with collision ionized equilibrium (CIE) plasma models. Evolved from early works in the 1970s, two CIE models are widely used nowadays for X-ray spectral analysis of the ICM: APEC [12] and cie in SPEX ${ }^{2}$ [13].

\footnotetext{
${ }^{1} \mathrm{~A}$ smaller aggregate of galaxies is referred to as a galaxy group.

${ }^{2} \mathrm{SPEX}$ is a spectral analysis package, which contains plasma models like cie (for collisional ionized plasmas) and pion (for photoionized plasmas). APEC is a standalone plasma model that can be used by spectral analysis packages like XSPEC [14].
} 


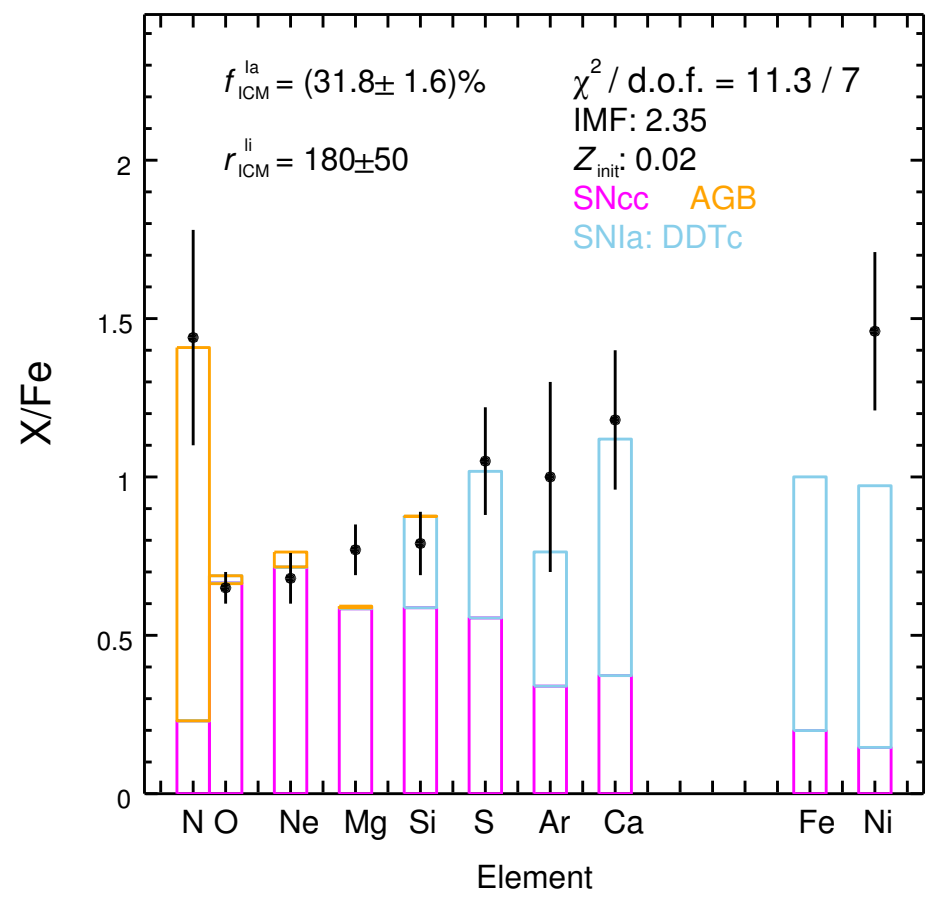

Figure 1. The chemical enrichment of the hot plasma in NGC 5044 observed with XMM-Newton. The abundance ratios are measured with the cie model in SPEX v3.04. Under a standard initial mass function (IMF) with a slope of $-2.35[10]$ and an initial metallicity $\left(Z_{\text {init }}=0.02\right.$, i.e. solar composition) of progenitors, a suitable mixture of different enrichment channels are required to fit the observed abundance ratios. Nitrogen is mainly enriched via the stellar winds of AGB stars. Core-collapse supernovae (SNcc) are the main elemental factory of the so-called $\alpha$-elements (e.g., $\mathrm{O}, \mathrm{Ne}$, and $\mathrm{Mg}$ ). Type Ia supernovae (SNIa) dominate the enrichment of the Fe-peak elements (e.g., Fe and Ni). This figure, originally shown in [11], is reproduced with permission.

Nevertheless, those pre-launch versions of APEC (v3.02) and SPEX (v3.00) do not provide a good fit to the newly obtained Perseus spectrum by Hitomi in 2016. The mismatch triggers the implementation of some necessary updates, including extending incomplete atomic database, replacing less accurate atomic data, and improving the level population calculation [15]. Updated plasma models not only better fit the observed Hitomi spectra of Perseus [15], but also lead to a revised interpretation of spectra observed with CCD type of spectrometers ${ }^{3}$ like the European Photo Imaging Camera (EPIC) aboard XMM-Newton [16].

Prior to the launch of Hitomi, 44 nearby relaxed galaxy groups and clusters were observed with XMM-Newton to study their chemical composition and origin (referred to as the CHEERS sample hereafter) [17]. The cie model in SPEX v2.06 was used to fit the EPIC spectra of the CHEERS sample [18]. Abundance ratios with respect to Fe were obtained and used to infer the

\footnotetext{
${ }^{3}$ Generally speaking, the spectral resolution of microcalorimeters $(<5 \mathrm{eV})$ is at least an order of magnitude better than that of CCD spectrometers $(50-200 \mathrm{eV})$.
} 
origin of the elements [19]. Later, the updated cie model in SPEX v3.04 was available, [16] reestimate the abundance ratios of the CHEERS sample. Table 1 presents the comparison between the two abundance measurements. While the rest of the abundance ratios are consistent within uncertainties between the two versions of plasma code, the $\mathrm{Cr} / \mathrm{Fe}$ and $\mathrm{Ni} / \mathrm{Fe}$ ratios are closer to unity (i.e. protosolar abundance ratio) in the new version. This is mainly due to the fact that the new plasma model includes hundreds of thousands of weak lines.

Table 1. Averaged abundance ratios with respect to Fe for the CHEERS sample. The original measurements were obtained with SPEX v2.06 as reported in [18]. The new version of SPEX (v3.04) was used by [16] to re-estimate the abundance ratios.

\begin{tabular}{|c|c|c||c|c|c|}
\hline Ratio & SPEX v2.06 & SPEX v3.04 & Ratio & SPEX v2.06 & SPEX v3.04 \\
\hline $\mathrm{O} / \mathrm{Fe}$ & $0.82 \pm 0.15$ & $0.82 \pm 0.18$ & $\mathrm{Ne} / \mathrm{Fe}$ & $0.72 \pm 0.16$ & $0.72 \pm 0.13$ \\
$\mathrm{Mg} / \mathrm{Fe}$ & $0.74 \pm 0.17$ & $0.94 \pm 0.07$ & $\mathrm{Si} / \mathrm{Fe}$ & $0.87 \pm 0.06$ & $0.95 \pm 0.06$ \\
$\mathrm{~S} / \mathrm{Fe}$ & $0.98 \pm 0.10$ & $1.00 \pm 0.02$ & $\mathrm{Ar} / \mathrm{Fe}$ & $0.88 \pm 0.15$ & $0.98 \pm 0.08$ \\
$\mathrm{Ca} / \mathrm{Fe}$ & $1.22 \pm 0.10$ & $1.27 \pm 0.10$ & $\mathrm{Cr} / \mathrm{Fe}$ & $1.56 \pm 0.19$ & $0.99 \pm 0.19$ \\
$\mathrm{Mn} / \mathrm{Fe}$ & $1.70 \pm 0.22$ & $1.56 \pm 0.77$ & $\mathrm{Ni} / \mathrm{Fe}$ & $1.93 \pm 0.40$ & $0.96 \pm 0.38$ \\
\hline
\end{tabular}

After the revision, the solar abundance ratios in the CHEERS sample (observed with XMMNewton/EPIC) agree with measurements from the Hitomi/SXS spectrum of Perseus [20, 22], indicating a common solar composition of the hot plasmas in the core regions of nearby relaxed groups and clusters of galaxies [16]. In addition, setting aside the uncertainties in the chemical enrichment models and nucleosynthesis yields [22], the new abundance ratios suggest that nearChandrasekhar-mass type Ia supernovae contribute significantly to the observed solar abundance ratios [21].

\section{Density of photoionized outflows}

At the center of almost every galaxy but the smallest lies a supermassive black hole (SMBH) with $M_{\mathrm{BH}}>10^{5} \mathrm{M}_{\odot}$ [23]. The growth of the $\mathrm{SMBH}$ is realized via accretion of matter. When the accretion rate is above a certain limit ( $\gtrsim 10^{-5}$ Eddington ratio), the central region of the galaxy is called an active galactic nucleus (AGN) [23].

While AGNs are powered by accretion (or inflows), outflows transporting matter and energy away from the nucleus to the host galaxy and beyond are also observed [24]. More importantly, the discovery of the tight correlation between the mass of SMBH and the observables (velocity dispersion and K-band luminosity) of the bulge component of the host galaxy [25] suggest that AGN-driven outflows might be the key to understand the evolution of SMBH and host galaxy [26-29].

Various forms of AGN outflows have been observed in the past two decades [30, 31]. In the $\mathrm{X}$-ray band, outflows have been observed in many active galaxies [32, 33]. Nevertheless, it is rather uncertain whether these X-ray outflows are powerful enough to play an important role in the interplay between the SMBH and its host galaxy.

The kinetic power carried by X-ray outflows can be estimated if the distance of the outflow with respect to the SMBH is known. Direct measurement of the outflow distance with respect to 
the black hole is not available. The size scale of SMBH is too small and the host galaxies are too far away so that it cannot be resolved via direct imaging.

Density sensitive metastable absorption lines can be used to measure the density of the outflows. In a low density (photoionized) plasma, the level population of each ion is concentrated in the ground level, so that only absorption lines from the ground levels can be observed. As the plasma density increases, the collision between the free electrons and ions can populate the metastable levels, which leads to the presence of metastable absorption line(s) in the spectrum. As the total level population is conserved for each ion, the strong the metastable absorption line(s), the weaker the ground absorption line.

For observers, a spectrometer with a sufficient spectral resolution and an adequate photon collecting area is essential. The former ensures that the metastable level absorption lines are clearly separated from the ground level absorption lines, ideally with both line profiles resolved. The latter ensures a high signal to noise ratio of the absorption features with reasonable exposure (up to a few hundreds of kilo-seconds). The current generation of grating spectrometers [36-38] are not very effective for the density measurement with metastable level absorption lines. While a tight constrain on the density of a disk wind in a stellar mass black hole GRO J1655-40 [39], only upper or lower limits are obtained for densities of AGN outflows[40-42].

On the other hand, the density measurement relies on a photoionized plasma model built on an extensive atomic database. Inaccurate metastable to ground level population ratio can lead to biased estimations of density, thus, distance and kinetic power of AGN outflows. Prior to the release of the pion model in SPEX v3.04, the collisional ionized equilibrium (CIE) model in the CHIANTI code was widely used to estimate the metastable to ground level population ratio. An assumption of the temperature of the CIE plasma is required to mimic the ionization balance of the photoionized outflow. Moreover, metastable levels and lines are not fully resolved in those early versions of CHIANTI. For instance, in the study of the ionized outflow in NGC 4051, [41] used CHIANTI v6 [43] to estimate the ratio of the equivalent width of the metastable absorption line at $12.38 \AA$ to the ground absorption line at $12.29 \AA$ of C-like Fe XXI. In fact, for C-like ions (including Fe XXI), three metastable absorption lines can be observed (provided with adequate spectral resolution) from three metastable levels $\left(2 s^{2} 2 p^{2},{ }^{3} P_{1},{ }^{3} P_{2}\right.$, and $\left.{ }^{3} D_{2}\right)$. For Fe XXI, the most prominent metastable absorption lines are $12.433 \AA$ (with the oscillator strength $f=0.43$ ), $12.331 \AA(f=0.59$ ), and $12.411 \AA(f=0.94)$ [42]. Moreover, the population of one of the metastable levels is sensitive to both the density and ionization parameter (or temperature) of the plasmas (Figure 2). A re-analysis of the ionized outflow in NGC 4051 is under review.

\section{Future perspectives}

The high-resolution X-ray observations of Perseus galaxy cluster with Hitomi/SXS certainly helped to identify issues with pre-launch plasma models, as well as the accuracy of the underlying atomic data. Note that the benchmark was limited to spectral features above $1.8 \mathrm{keV}$, because a 262micron Be-like filter was used to block soft X-ray photons during the performance validating phase. Unfortunately, Hitomi spun out of control less than two months since its launch date. Several Hitomi-level missions (XRISM, Athena [44], Arcus [45], and HUBS, etc.) have been proposed and some funded. 


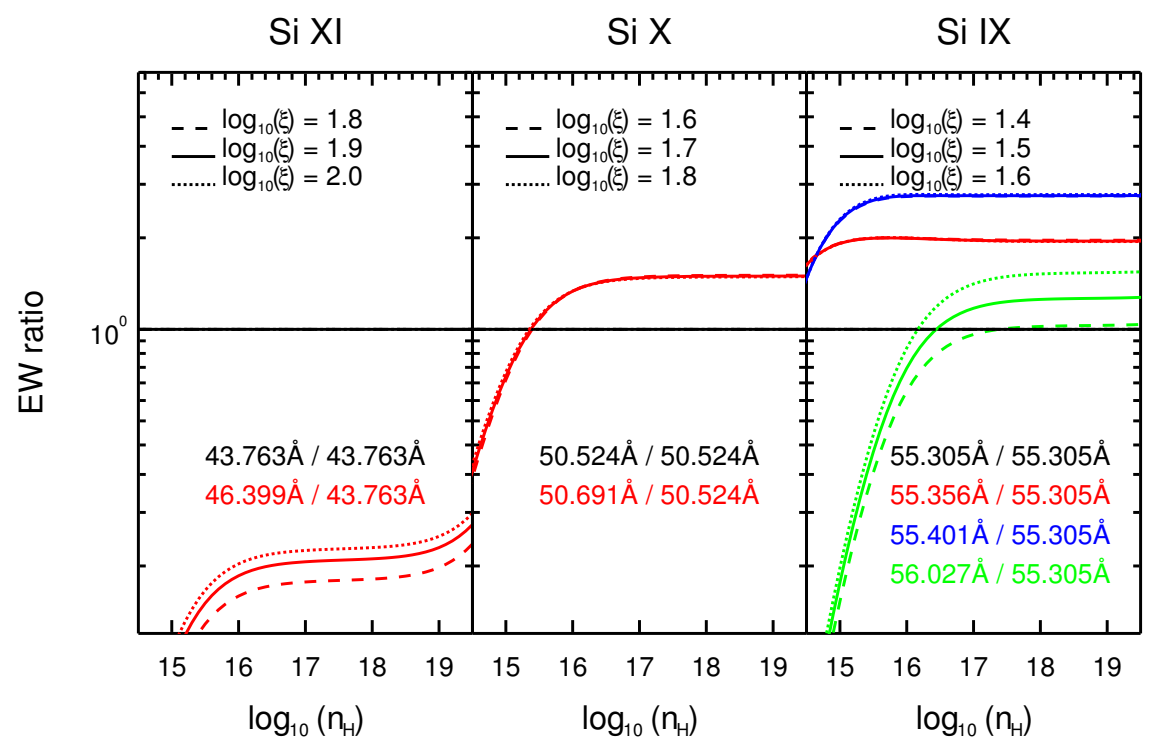

Figure 2. The equivalent width (EW) ratio of the metastable level absorption line to the ground level absorption line as a function of plasma density $\left(n_{\mathrm{H}}\right)$ for (Be-like) Si XI, (B-like) Si X, and (C-like) Si IX [42]. Different line styles indicate different ionization parameter $(\xi)$ of the photoionized plasma.

Plasma models need to be prepared for the high-quality X-ray spectra in the near future. Some efforts have been initiated in the community. The first one is to recognize the incompleteness of the current atomic databases. Level-resolved rates and cross-sections spanning several orders of magnitude in the parameter space (e.g. temperature and density) are the building blocks of the plasma model. Therefore, the number of levels for each ion included in a plasma model indicates the (in)completeness of atomic data. An impression of the number of energy levels for each ion included in the atomic database of SPEX v3.05 is illustrated in Figure 3. Generally speaking, elements with a even atomic number $(Z)$ have more data as they are more abundant in astrophysical environments. Compared to $\mathrm{H}$-like to B-like ions, atomic data of ions with six or more electrons are lacking, except for $\mathrm{Fe}$ and $\mathrm{Ni}$ ions.

A similar level of incompleteness applies to the atomic database of other codes like APEC and CHIANTI. Furthermore, detailed rates, cross-sections and sometimes energy levels can differ significantly among these atomic databases. Without being complete, we list a few sources of discrepancy. First, the existence of outdated atomic data while recent and accurate atomic data are available. Second, some atomic data are only accurate in a limited range of parameter space but extrapolation can sometimes lead to undesired behavior. Third, several theoretical calculations might be available, yet do not agree with each other. Due to the lack of benchmarking with lab measurements, different plasma codes and models might take different calculations. Therefore, comparisons among different plasma models and codes have been made [15, 46] to identify the discrepancy, especially those related to important transitions.

To advance our knowledge of the Universe, better plasma models and atomic data are certainly required. Odd- $Z$ elements are important, for instance, their abundance are more sensitive to the initial metallicity of the progenitors of AGBs and SNe [11], which helps us to better understand the 


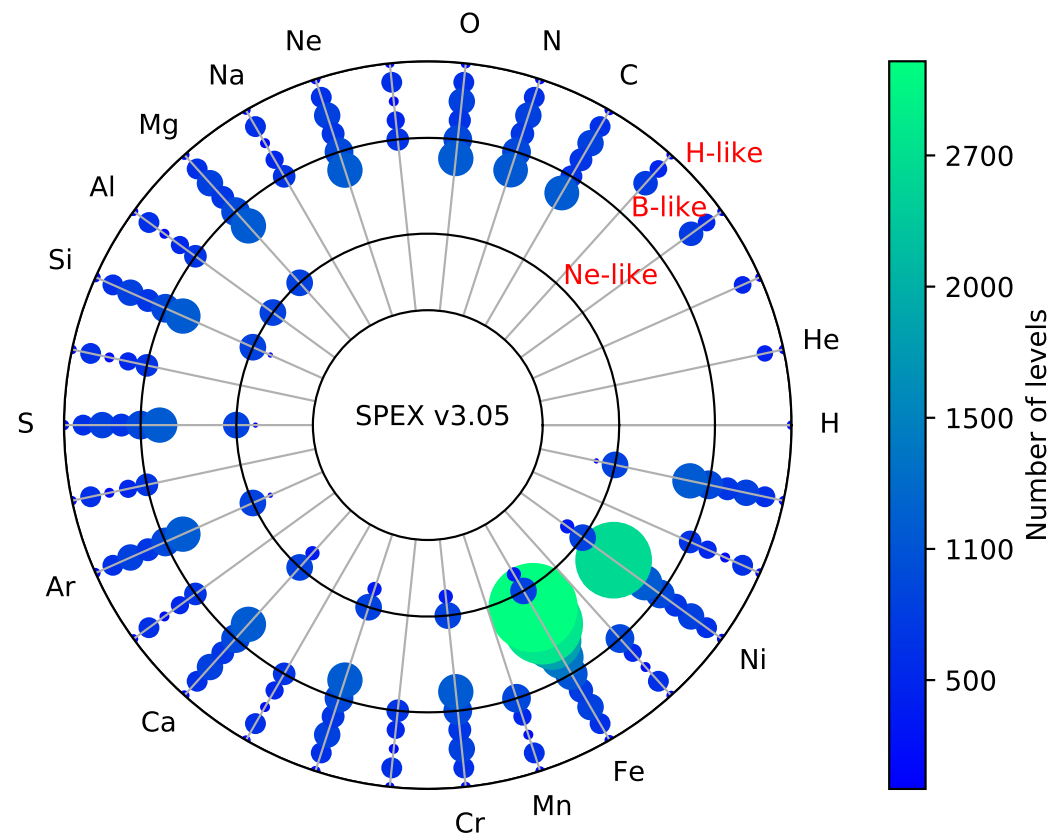

Figure 3. The number of energy levels for each ion (color coded) included in the latest version of the SPEX code (v3.05). The (in)completeness can also be inferred from the size of the filled circle. Many level-resolved rates and cross-sections are available for $\mathrm{H}$-like (outer most ring) to B-like ions with atomic number with $Z \leq 30$. Atomic data for C-like, Ne-like and Na-like ions are limited to cosmic abundance elements (e.g., C, $\mathrm{N}, \mathrm{O}, \mathrm{Si}, \mathrm{Fe}$, and Ni). Data for N-like to F-like data are lacking, except for Fe and Ni ions.

chemical enrichment of the Universe. The ground and metastable absorption lines of various ions are essential to probe AGN outflows in a wide range of density and distance [42]. The accuracy of plasma models correspond to spectral features below $1.8 \mathrm{keV}$ (not observed with Hitomi/SXS) needs to be investigated. The Fe abundance of low-temperature (and low-mass) elliptical galaxies and groups of galaxies is measured from the Fe-L band emission features below $1.8 \mathrm{keV}$. Two different trends of iron abundance with an increasing temperature/mass are reported using different (versions of) plasma models [47, 48], which requires re-investigation with the future plasma codes. The above examples are just a taste of the need of plasma models and atomic data.

Once we know our need for atomic data, theoreticians can perform new calculations to complete the atomic database or improve the accuracy of the existing data $[49,50]$, new lab measurements can be triggered to benchmark theoretical calculations [51]. Subsequently, modellers need to take advantage of more accurate atomic data and validate the implementation (e.g., parameterization, interpolation, and extrapolation) in the plasma models. The remaining discrepancy among different plasma models and codes can be used as a measure of systematic uncertainty.

\section{Acknowledgments}

J.M. is supported by STFC (UK) through the University of Strathclyde UK APAP network grant ST/R000743/1. L.G. is supported by the RIKEN Special Postdoctoral Researcher Program. F. M. is supported by the Lendület LP2016-11 grant awarded by the Hungarian Academy of Sciences. M.M. 
is supported by NWO through the Innovational Research Incentives Scheme Vidi grant 639.042.525. SRON is supported financially by NWO, the Netherlands Organization for Scientific Research.

\section{References}

[1] Mitchell, R. J., Culhane, J. L., Davison, P. J. N., et al., Ariel 5 observations of the X-ray spectrum of the Perseus cluster, Mon. Not. R. Astron. Soc. 175 (1976) 29.

[2] Takahashi, T., Mitsuda, K., Kelley, R., et al., The ASTRO-H X-ray astronomy satellite, Space Telescopes and Instrumentation 2014: Ultraviolet to Gamma Ray (2014) 914425.

[3] Hitomi Collaboration, The quiescent intracluster medium in the core of the Perseus cluster, Nature 535 (2016) 117.

[4] Kaastra, J. S., Gu, L., Mao, J., et al., Science with hot astrophysical plasmas, J. Instrum. 12 (2017) C08008.

[5] Schindler, S., \& Diaferio, A., Metal Enrichment Processes, Space Sci. Rev. 134 (2008) 363.

[6] Anderson, M. E., \& Bregman, J. N., Do Hot Halos Around Galaxies Contain the Missing Baryons?, Astrophys. J. 714 (2010) 320.

[7] Budzynski, J. M., Koposov, S. E., McCarthy, I. G., et al., Mon. Not. R. Astron. Soc., 437 (2014) 1362.

[8] Karakas, A. I., Updated stellar yields from asymptotic giant branch models, Mon. Not. R. Astron. Soc. 403 (2010) 1413.

[9] Nomoto, K., Kobayashi, C., \& Tominaga, N., Nucleosynthesis in Stars and the Chemical Enrichment of Galaxies, Annu. Rev. Astron. Astr. 51 (2013) 457.

[10] Salpeter, E. E., The Luminosity Function and Stellar Evolution., Astrophys. J. 121 (1955) 161.

[11] Mao, J., de Plaa, J., Kaastra, J. S., et al., Nitrogen abundance in the X-ray halos of clusters and groups of galaxies, Astron. Astrophys. 621 (2019) A9.

[12] Smith, R. K., Brickhouse, N. S., Liedahl, D. A., et al., Collisional Plasma Models with APEC/APED: Emission-Line Diagnostics of Hydrogen-like and Helium-like Ions, Astrophys. J. 556 (2001) L91.

[13] Kaastra, J. S., Mewe, R., \& Nieuwenhuijzen, H., SPEX: a new code for spectral analysis of X \& UV spectra., UV and X-ray Spectroscopy of Astrophysical and Laboratory Plasmas (1996) 411.

[14] Arnaud, K. A., XSPEC: The First Ten Years, Astronomical Data Analysis Software and Systems V (1996) 17.

[15] Hitomi Collaboration Atomic data and spectral modeling constraints from high-resolution X-ray observations of the Perseus cluster with Hitomi, Publ. Astron. Soc. Jpn., 70 (2018) 12.

[16] Mernier, F., Werner, N., de Plaa, J., et al., Solar chemical composition in the hot gas of cool-core ellipticals, groups, and clusters of galaxies, Mon. Not. R. Astron. Soc. 480 (2018) L95.

[17] de Plaa, J., Kaastra, J. S., Werner, N., et al., CHEERS: The chemical evolution RGS sample, Astron. Astrophys. 607 (2017) A98.

[18] Mernier, F., de Plaa, J., Pinto, C., et al., Origin of central abundances in the hot intra-cluster medium. I. Individual and average abundance ratios from XMM-Newton EPIC, Astron. Astrophys. 592 (2016) A157.

[19] Mernier, F., de Plaa, J., Pinto, C., et al., Origin of central abundances in the hot intra-cluster medium. II. Chemical enrichment and supernova yield models, Astron. Astrophys. 595 (2016) A126. 
[20] Hitomi Collaboration, Solar abundance ratios of the iron-peak elements in the Perseus cluster, Nature 551 (2017) 478.

[21] Mernier, F., Biffi, V., Yamaguchi, H., et al., Enrichment of the Hot Intracluster Medium: Observations, Space Sci. Rev. 214 (2018) 129.

[22] Simionescu, A., Nakashima, S., Yamaguchi, H., et al., Constraints on the chemical enrichment history of the Perseus Cluster of galaxies from high-resolution X-ray spectroscopy, Mon. Not. R. Astron. Soc. 483 (2019) 1701.

[23] Netzer, H., Revisiting the Unified Model of Active Galactic Nuclei, Annu. Rev. Astron. Astr. 53 (2015) 365.

[24] Crenshaw, D. M., Kraemer, S. B., \& George, I. M., Mass Loss from the Nuclei of Active Galaxies, Annu. Rev. Astron. Astr. 41 (2003) 117.

[25] Kormendy, J., \& Ho, L. C., Coevolution (Or Not) of Supermassive Black Holes and Host Galaxies, Annu. Rev. Astron. Astr. 51 (2013) 511.

[26] Magorrian, J., Tremaine, S., Richstone, D., et al., The Demography of Massive Dark Objects in Galaxy Centers, Astron. J., 115 (1998) 2285.

[27] Silk, J., \& Rees, M. J., Quasars and galaxy formation, Astron. Astrophys. 331 (1998) L1.

[28] Lakhchaura, K., Truong, N., \& Werner, N., Correlations between supermassive black holes, hot atmospheres, and the total masses of early type galaxies (2019), arXiv:1904.10513.

[29] Gaspari, M., Eckert, D., Ettori, S., et al., The X-ray Halo Scaling Relations of Supermassive Black Holes, (2019) arXiv:1904.10972.

[30] Fabian, A. C.Observational Evidence of Active Galactic Nuclei Feedback, Annu. Rev. Astron. Astr. 50 (2012) 455.

[31] Harrison, C. M., Costa, T., Tadhunter, C. N., et al., AGN outflows and feedback twenty years on, Nat. Astron. 2 (2018) 198.

[32] Tombesi, F., Cappi, M., Reeves, J. N., et al., Unification of X-ray winds in Seyfert galaxies: from ultra-fast outflows to warm absorbers Mon. Not. R. Astron. Soc. 430 (2013) 1102.

[33] Laha, S., Guainazzi, M., Dewangan, G. C., et al., Warm absorbers in X-rays (WAX), a comprehensive high-resolution grating spectral study of a sample of Seyfert galaxies - I. A global view and frequency of occurrence of warm absorbers., Mon. Not. R. Astron. Soc. 441 (2014) 2613.

[34] Tarter, C. B., Tucker, W. H., \& Salpeter, E. E., The Interaction of X-Ray Sources with Optically Thin Environments, Astrophys. J. 156 (1969) 943.

[35] Krolik, J. H., McKee, C. F., \& Tarter, C. B., Two-phase models of quasar emission line regions., Astrophys. J. 249 (1981) 422.

[36] Brinkman, A. C., Gunsing, C. J. T., Kaastra, J. S., et al., First Light Measurements of Capella with the Low-Energy Transmission Grating Spectrometer aboard the Chandra X-Ray Observatory, Astrophys. J. 530 (2000) L111.

[37] den Herder, J. W., Brinkman, A. C., Kahn, S. M., et al., The Reflection Grating Spectrometer on board XMM-Newton, Astron. Astrophys. 365 (2001) L7.

[38] Canizares, C. R., Davis, J. E., Dewey, D., et al., The Chandra High-Energy Transmission Grating: Design, Fabrication, Ground Calibration, and 5 Years in Flight, Publications of the Astronomical Society of the Pacific 117 (2005) 1144. 
[39] Miller, J. M., Raymond, J., Reynolds, C. S., et al., The Accretion Disk Wind in the Black Hole GRO J1655-40, Astrophys. J. 680 (2008) 1359.

[40] Kaastra, J. S., Raassen, A. J. J., Mewe, R., et al., X-ray/UV campaign on the Mrk 279 outflow: Density diagnostics in Active Galactic Nuclei using $O V$ K-shell absorption lines, Astron. Astrophys., 428 (2004) 57.

[41] King, A. L., Miller, J. M., \& Raymond, J., Warm Absorbers and Outflows in the Seyfert-1 Galaxy NGC 4051, Astrophys. J. 746 (2012) 2.

[42] Mao, J., Kaastra, J. S., Mehdipour, M., et al., Density diagnostics of ionized outflows in active galactic nuclei. X-ray and UV absorption lines from metastable levels in Be-like to C-like ions, Astron. Astrophys. 607 (2017) A100.

[43] Dere, K. P., Landi, E., Young, P. R., et al., CHIANTI - an atomic database for emission lines. IX. Ionization rates, recombination rates, ionization equilibria for the elements hydrogen through zinc and updated atomic data, Astron. Astrophys. 498 (2009) 915.

[44] Nandra, K., Barret, D., Barcons, X., et al., The Hot and Energetic Universe: A White Paper presenting the science theme motivating the Athena+ mission, (2013) arXiv:1306.2307.

[45] Smith, R. K., Abraham, M. H., Allured, R., et al., Arcus: the x-ray grating spectrometer explorer, Space Telescopes and Instrumentation 2016: Ultraviolet to Gamma Ray (2016) 99054M.

[46] Mehdipour, M., Kaastra, J. S., \& Kallman, T., Systematic comparison of photoionised plasma codes with application to spectroscopic studies of AGN in X-rays, Astron. Astrophys. 596 (2016) A65.

[47] Yates, R. M., Thomas, P. A., \& Henriques, B. M. B., Iron in galaxy groups and clusters: confronting galaxy evolution models with a newly homogenized data set, Mon. Not. R. Astron. Soc., 464 (2017) 3169.

[48] Mernier, F., Werner, N., de Plaa, J., et al., Mass-invariance of the iron enrichment in the hot haloes of massive ellipticals, groups, and clusters of galaxies, Mon. Not. R. Astron. Soc. 480 (2018) L95.

[49] Mao, J., Kaastra, J., \& Badnell, N. R., The electron energy loss rate due to radiative recombination, Astron. Astrophys. 599 (2017) A10.

[50] Gu, L. Y., Raassen, A. J. J., Mao, J., et al., X-ray spectra of the Fe-L complex, (2019) arXiv:1905.07871.

[51] Shah, C., Crespo López-Urrutia, J. R., Gu, M. F., et al., Revisiting the Fe XVII line emission problem: High-resolution laboratory measurements of the $3 s-2 p$ and $3 d-2 p$ line-formation channels, (2019) arXiv:1903.04506. 